\title{
A PARALLEL ADAPTIVE 3D MHD SCHEME FOR MODELING CORONAL AND SOLAR WIND PLASMA FLOWS
}

\author{
C. P. T. GROTH, D. L. DE ZEEUW and T. I. GOMBOSI \\ Space Physics Research Laboratory, Department of Atmospheric, Oceanic and Space Sciences, \\ University of Michigan, Ann Arbor, MI 48109-2143, USA \\ K. G. POWELL \\ Department of Aerospace Engineering, University of Michigan, Ann Arbor, MI 48109-2118, USA
}

\begin{abstract}
A parallel adaptive mesh refinement (AMR) scheme is described for solving the governing equations of ideal magnetohydrodynamics (MHD) in three space dimensions. This solution algorithm makes use of modern finite-volume numerical methodology to provide a combination of high solution accuracy and computational robustness. Efficient and scalable implementations of the method have been developed for massively parallel computer architectures and high performance achieved. Numerical results are discussed for a simplified model of the initiation and evolution of coronal mass ejections (CMEs) in the inner heliosphere. The results demonstrate the potential of this numerical tool for enhancing our understanding of coronal and solar wind plasma processes.
\end{abstract}

Key words: magnetohydrodynamics, parallel computing, adaptive mesh refinement, solar wind, coronal mass ejection

\section{Introduction}

Global models based on the numerical solution of the equations of magnetohydrodynamics (MHD) represent a very important component of efforts to understand coronal and solar wind plasma flows. While providing only a low-order approximation to the behavior of conducting fluids, MHD models have been used successfully to simulate and advance our understanding of many space plasma processes. Examples of the application of MHD models to the study of coronal and solar wind plasma flows include the studies by Mikić and Linker (1994), Linker and Mikić (1995), Wu and Guo (1997), Guo and Wu (1998), Lionello et al. (1998), Dryer (1998), and Odstrčil and Pizzo (1998). In this paper, the application of a new parallel solution-adaptive MHD model to the simulation of fully three-dimensional solar wind plasma flows is described. Numerical results are discussed for a coronal mass ejection (CME) driven by a local plasma density enhancement in order to demonstrate the potential of the approach.

\section{Parallel Solution-Adaptive 3D MHD Model}

In the last decade, several developments have led to a new paradigm for the numerical solution of partial differential equations (PDEs). They are: (1) advances in numerical methods, particularly for hyperbolic conservation laws, (2) the evolution of solution-adaptive techniques from a research topic to a practical tool, and (3) the advent of massively parallel computers. By capitalizing on these developments, a new, efficient, and reliable parallel solution-adaptive algorithm has been developed 
for solving the hyperbolic PDEs of ideal MHD in three space dimensions. The main elements of this algorithm are now briefly outlined.

\subsection{UPWIND FINITE-VOLUME SCHEME}

A cell-centered upwind finite-volume formulation of the type first proposed by Godunov (1959) is adopted to solve the standard eight equations of ideal MHD in weak conservation or divergence form (Powell 1994; Powell et al. 1995).* Two of the more popular approximate Riemann solvers - the Riemann solver due to Roe (1981) and a modified version of the method of Harten, Lax, Van Leer, and Einfeldt (Harten et al. 1983; Einfeldt et al. 1991) —are employed in the evaluation of the numerical flux. Originally devised for gas dynamics, these flux functions have been rederived for MHD to cope with the solenoidal condition and deal with degeneracies in the MHD eigenstructure (Powell 1994; Powell et al. 1995; Roe and Balsara 1996; Linde 1998). Limited linear reconstruction is used to achieve higherorder accuracy (Van Leer 1979) and multi-stage schemes (Van Leer et al. 1989), with point-implicit treatment of source terms, are used to integrate the ordinary differential equations that result from the spatial discretization of the governing PDEs. The finite-volume scheme solves the hydrodynamic and magnetic equations in a tightly coupled manner, provides accurate resolution of discontinuities, and works equally well across a range of plasma $\beta$, where $\beta$ is the ratio of thermal and magnetic pressures.

\subsection{BLOCK-BASEd AdAPTIVE MESH REFINEMENT}

Computational grids that automatically adapt to the solution of the governing PDEs are effective in treating problems with multiple length scales, greatly reducing the computing resources required for many problems. Borrowing form previous work by Berger (Berger 1984; Berger and Colella 1989), Quirk (Quirk 1991; Quirk and Hanebutte 1993), and De Zeeuw and Powell (1993) and keeping in mind the desire for high performance on parallel architectures, a block-based adaptive mesh refinement (AMR) technique has been developed for the preceding upwind finitevolume formulation. In this approach, the governing equations are integrated to obtain volume-averaged solution quantities within rectangular Cartesian computational cells. The cells are embedded in regular structured blocks with cells of equal size. The blocks are self-similar and all consist of $N_{x} \times N_{y} \times N_{z}$ cells. Computational grids are then taken to be composed of many blocks and mesh adaption is accomplished by dividing and coarsening the appropriate blocks, with changes in cell resolution by a factor of two permitted between adjacent blocks. In regions

\footnotetext{
* For the applications of interest here, the MHD equations are supplemented with additional source terms representing the effects of the solar gravitational force and plasma heating. In the case of the latter, an empirically formulated volumetric heating function is included to model micro-physical processes not represented by the ideal MHD description, such as coronal heating and heat transfer effects.
} 

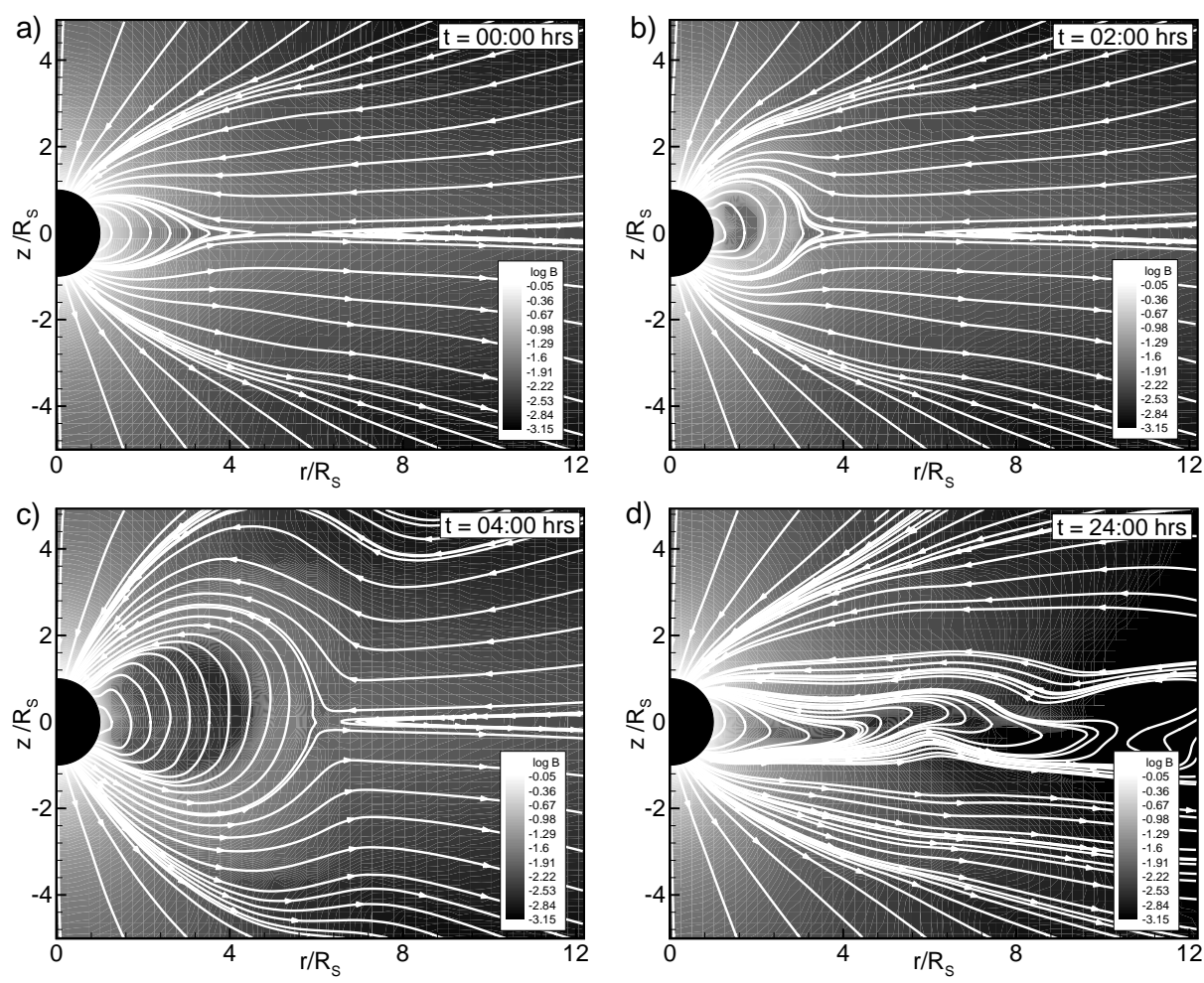

Figure 1. Four meridional snapshots of the computed CME solution depicting the initiation and evolution of the disturbance from $t=0$ hours to $t=24$ hours.

that require increased resolution, a block is refined by dividing, with each of its eight octants becoming a block having the same number of cells as the original. In regions that are deemed over-resolved, the refinement process is reversed and eight blocks are coarsened and coalesced into a single block with half the spatial resolution. Physics-based refinement criteria are used to direct coarsening and division of blocks. A hierarchical data structure is used to track mesh refinement and solution block connectivity (De Zeeuw et al. 1998).

\subsection{PARALLEL IMPLEMENTATION}

The preceding solution-adaptive scheme is designed to achieve high performance on massively parallel architectures. Parallel implementation of the algorithm has been carried out using FORTRAN 90 and the message passing interface (MPI) library on several multi-processor platforms, and 233 GFlops has been attained on a 1,024-processor Cray T3E with near-perfect scalability (De Zeeuw et al. 1998). 


\section{Simulation of Coronal Mass Ejections}

Some initial studies of the formation and evolution of CMEs have been carried out using the parallel solution-adaptive MHD scheme described above. In these studies, a model of the nominal background solar wind for conditions during solar minimum was used to represent the initial state of the solar wind, and CMEs were initiated by localized isothermal density and pressure enhancements. While the magnetic field configuration and $\mathrm{CME}$ onset mechanism used in the simulations were somewhat oversimplified, these calculations represent initial steps towards developing more sophisticated models of the solar wind.

In the CME simulations, the "steady-state" solar wind was modeled by assuming that the inner solar corona is a large rotating reservoir of hot plasma with an embedded dipole field. The reservoir temperature and number density were taken to be $3.1 \mathrm{MK}$ and $10^{8} \mathrm{~cm}^{-3}$, respectively, and the strength of the magnetic field at the poles was $1 \mathrm{G}$. A value of $5 / 3$ was used for the specific heat ratio such that adiabatic cooling at large heliocentric distances was correctly modeled. The coronal plasma was heated by volumetric heating in the vicinity of the Sun where the latitude and radial dependence of the heat source was chosen to produce a reasonable solar wind, having both fast and slow streams.

Figure 1a shows a meridional cut through the calculated initial solar wind solution. The shading represents the logarithm of the magnitude of the magnetic field and the white lines correspond to field lines. It is evident that the solution, which is dictated by a complex balance between pressure, magnetic, gravitational, and inertial forces, has regions of open and closed field lines and is characterized by the now classical "helmet" streamer configuration with associated neutral point and equatorial current sheet similar to that obtained by Pneuman and Kopp (1971). However, unlike the Pneuman-Kopp model, the solution more correctly mimics the two-state nature of the solar wind (McComas et al. 1998). Fast solar wind (800 $\mathrm{km} / \mathrm{s}$ ) originating from polar coronal holes is produced above $30^{\circ}$ in heliolatitude, slow solar wind $(400 \mathrm{~km} / \mathrm{s})$ is produced near the solar equator at lower latitudes, and reasonable values for the plasma properties and interplanetary magnetic field are obtained at 1/2 AU. Refer to Figure 2. Shown is a polar diagram of the asymptotic values of the computed flow speed as a function of heliolatitude. The numerical solution is consistent with Ulysses/SWOOPS observations (McComas et al. 1998), also shown in the figure.

Using the preceding initial state, numerical results for a CME driven by an isothermal density enhancement are shown in Figures $1 \mathrm{~b}-\mathrm{d}$. The solution at times $t=2,4$, and 24 hours after onset are shown. For this calculation, a 40:1 density enhancement was introduced with a duration of 16 hours at the solar surface just above the equatorial plane. The density enhancement first leads to the "filling" of closed magnetic field lines with additional plasma. This is followed by an expansion of the closed field line region. After a period of time, the closed field lines are unable to contain additional plasma and the streamer belt is disrupted. The resulting CME 


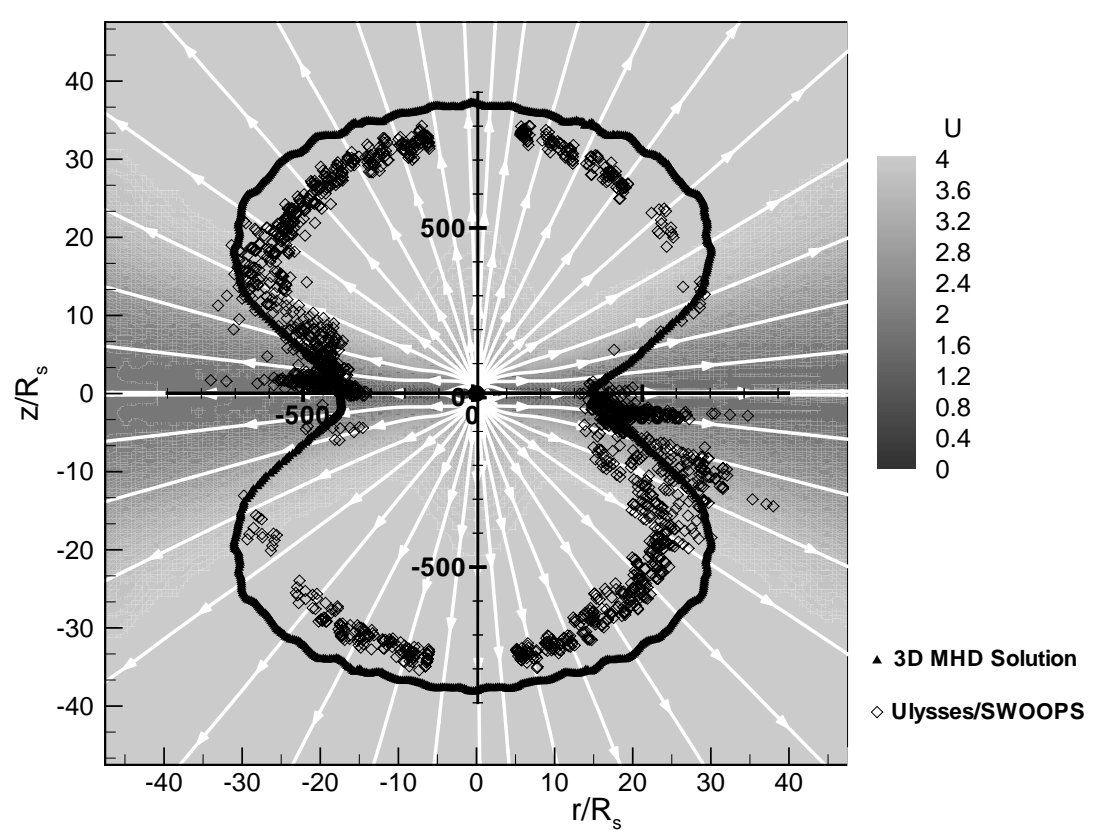

Figure 2. Predicted solar wind velocity and stream lines, and polar plot of computed solar wind speed compared to Ulysses/SWOOPS data (McComas et al. 1998).

moves rapidly through the inner corona and propagates outward, dragging out closed field lines with it and disrupting the heliospheric current sheet as it moves. A magnetic cavity propagates behind the front of the disturbance, which moves at velocities nearing $450 \mathrm{~km} / \mathrm{s}$. Between $t=17$ and $t=19$ hours, the density enhancement completely diminishes and CME field lines begin disconnecting from the solar surface. This results in the reformation of the current sheet. The solution at $t=24$ hours of Figure 1d depicts this reformation process.

This work was supported by the NSF-NASA-AFOSR inter-agency grant NSF ATM-9318181 and by NASA HPCC CAN NCCS5-146.

\section{References}

Berger, M. J.: 1984, Adaptive mesh refinement for hyperbolic partial differential equations, J. Comput. Phys., 53, 484.

Berger, M. J., and Colella, P.: 1989, Local adaptive mesh refinement for shock hydrodynamics, J. Comput. Phys., 82, 67.

De Zeeuw, D., and Powell, K. G.: 1993, An adaptively refined Cartesian mesh solver for the Euler equations, J. Comput. Phys., 104, 56. 
De Zeeuw, D. L., Groth, C. P. T., Marshall, H. G., Gombosi, T. I., Powell, K. G., and Stout, Q. F.: 1998, A parallel adaptive high-resolution scheme for MHD with applications in space plasma physics, SIAM J. Sci. Comput., submitted.

Dryer, M.: 1998, Multidimensional MHD simulation of solar-generated disturbances: Space weather forecasting of geomagnetic storms, AIAA J., 36 (3), 365.

Einfeldt, B., Munz, C. D., Roe, P. L., and Sjögreen, B.: 1991, On Godunov-type methods near low densities, J. Comput. Phys., 92, 273.

Godunov, S. K.: 1959, Finite-difference method for numerical computations of discontinuous solutions of the equations of fluid dynamics, Mat. Sb., 47, 271.

Guo, W. P., and Wu, S. T.: 1998, A magnetohydrodynamic description of coronal helmet streamers containing a cavity, Astrophys. J., 494, 419.

Harten, A., Lax, P. D., and van Leer, B.: 1983, On upstream differencing and Godunov-type schemes for hyperbolic conservation laws, SIAM Rev., 25 (1), 35.

Linde, T. J.: 1998, A Three-Dimensional Adaptive Multifluid MHD Model of the Heliosphere, Ph.D. thesis, University of Michigan.

Linker, J. A., and Mikić, Z.: 1995, Disruption of a helmet streamer by photospheric shear, Astrophys. J., 438, L45.

Lionello, R., Mikić, Z., and Schnack, D. D.: 1998, Magnetohydrodynamics of solar coronal plasmas in cylindrical geometry, J. Comput. Phys., 140, 172.

McComas, D., et al.: Ulysses' return to the slow solar wind, Geophys. Res. Lett., 25, 1.

Mikić, Z., and Linker, J. A.: 1994, Disruption of coronal magnetic field arcades, Astrophys. J., 430, 898.

Odstrčil, D., and Pizzo, V. J.: 1998, Three-dimensional propagation of a CME within a tilted-dipole background solar wind flow, J. Geophys. Res., submitted.

Pneuman, G. W., and Kopp, R. A.: 1971, Gas-magnetic field interactions in the solar corona, Sol. Phys., 18, 258.

Powell, K. G.: 1994, An approximate Riemann solver for magnetohydrodynamics (that works in more than one dimension), Report 94-24, ICASE.

Powell, K. G., Roe, P. L., Myong, R. S., Gombosi, T. I., and De Zeeuw, D. L.: 1995, An upwind scheme for magnetohydrodynamics, Paper 95-1704-CP, AIAA.

Quirk, J. J.: 1991, An Adaptive Grid Algorithm for Computational Shock Hydrodynamics, Ph.D thesis, Cranfield Institute of Technology.

Quirk, J. J., and Hanebutte, U. R.: A parallel adaptive mesh refinement algorithm, Report 93-63, ICASE.

Roe, P. L.: 1981, Approximate Riemann solvers, parameter vectors, and difference schemes, J. Comput. Phys., 43, 357.

Roe, P. L., and Balsara, D. S.: 1996, Notes on the eigensystem of magnetohydrodynamics, SIAM J. Appl. Math., 56 (1), 57.

Van Leer, B.: 1979, Towards the ultimate conservative difference scheme. v. a second-order sequel to Godunov's method, J. Comput. Phys., 32, 101.

Van Leer, B., Tai, C. H., and Powell, K. G.: 1989, Design of optimally-smoothing multi-stage schemes for the Euler equations, Paper 89-1933-CP, AIAA.

Wu, S. T., and Guo, W. P.: 1997, A self-consistent numerical magnetohydrodynamic (MHD) model of helmet streamer and flux rope interactions: Initiation and propagation of coronal mass ejections (CMEs), in Coronal Mass Ejections, Geophysical Monograph 99, (American Geophysical Union, Washington). 